\title{
Criptococcose: revisão sistemática dos casos ocorridos no Brasil entre 1995-2005
}

\author{
Rodrigo dos Santos Diniz ${ }^{1}$ \\ Inara Dantas da Silva ${ }^{1}$ \\ Maria Tereza Barreto de Oliveira \\ Aurigena Antunes de Araújo Ferreira ${ }^{3}$
}

\begin{abstract}
Resumo
Neste artigo, apresenta-se uma revisão sistemática da literatura científica a respeito dos casos clínicos de criptococose ocorridos no Brasil. Foram selecionados trabalhos publicados na PUBMED, entre os anos de 1995 e 2005, totalizando 13 publicações, nas quais se encontram casos clínicos em pacientes com ou sem AIDS. Nove desses estudos ocorreram nas regiōes Centro-Oeste, Sul e Sudeste, e três deles envolvem a região Norte e Nordeste. Foram verificadas as seguintes variáveis: autor (es), ano, localidade, número de casos investigados, agente(s) etiológico(s), sinais e sintomas, doença isolada ou associada, resposta imune e desfecho. Pôde-se constatar que a maior parte dos casos relatados ocorreu como coinfecção associada a Aids, sendo o agente etiológico mais freqüente o Cryptococcus neoformans var. neoformans (sorotipos A e D). Esses casos foram encontrados com maior prevalência nas regiōes Sul, Sudeste e Centro-Oeste. A ocorrência de $C$ neoformans var. gattii (sorotipos $\mathrm{B}$ e C) está relacionada com áreas endêmicas, localizadas na região Norte e Nordeste. Os sinais e sintomas afetam, principalmente, o Sistema Nervoso Central e os pulmōes. Os casos relatados na literatura apresentam limitaçóes quanto ao desfecho da criptococose, possivelmente, devido ao fato de a patologia se desenvolver, principalmente, como uma co-infecção relacionada à Aids.
\end{abstract}

Palavras-chave: Cryptococcus neoformans; criptococose - literatura de revisão.

\section{INTRODUÇÃO}

A criptococcose é uma micose sistêmica oportunista, causada pelo Cryptococcus neoformans, um basidiomiceto que se apresenta em sua forma parasitária como levedura anamorfa capsulada. Está freqüentemente envolvido nos casos de meningoencefalite em pacientes com AIDS (CORRÊA et al., 1999; BOEKHOUT et al., 2001). Cryptococcus neoformans var. neoformans é um fungo cosmo- polita, que vive em solos contaminados com excretas de pombos ou de outras aves em regiós tropicais e de climas temperados. Os estudos relacionados a associação de Cryptococcus neoformans var. gattii ao Eucalyptus camaldulensis foi uma das grandes contribuições para a compreensão da ecologia desse microorganismo (SORREL et al., 1996). De acordo com a classificação atual, Cryptococcus neoformans possui

\footnotetext{
${ }^{1}$ Acadêmico do Curso de Farmácia. UFRN. Natal - RN

${ }^{2}$ Professora Adjunto de Microbiologia. UFRN. Natal - RN

${ }^{3}$ Professora Adjunto de Farmacologia. UFRN. Natal - RN

Correspondência para / Correspondence to:

Aurigena Antunes de Araújo Ferreira

Departamento de Biofísica e Farmacologia, Centro de Biociências - UFRN.

Av. Senador Salgado Filho, s.n. - Lagoa Nova - Campus Universitário.

59.075-000 Natal - Rio Grande do Norte - Brasil.

Tel.: (84)3215-3419

E-mail: aurigena@ufrnet.br
}

R. Ci. méd. biol., Salvador, v. 4, n. 3, p. 236-241, set./dez. 2005 
três variedades: $C$. neoformans (sorotipo A), var. grubii, Cryptococcus neoformans (sorotipo D), var. neoformans, sendo ambas variedades de ampla distribuição mundial, e Cryptococcus neoformans (sorotipo B e C), pertencentes à variedade gattii, que está limitada às regiōes tropicais e subtropicais do mundo. Ainda, um sorotipo híbrido $\mathrm{AD}$ pode ser reconhecido por técnicas sorológicas, moleculares de PCR, fazendo-se uso de primers específicos na caracterização dos mating-types, ou por caracterização de genótipos, usando-se a metodologia do AFLP (LENGELER; COX; HEITMAN, 2001; OLIVEIRA et al., 2004). O Cryptococcus neoformans var. gattii tem sido isolado mais freqüentemente de indivíduos imunocompetentes, ao passo que C. neoformans var. neoformans está fortemente associado com estados de imunodeficiência entre indivíduos com AIDS (VERONESI; FOCACCIA, 1996).

Os fatores implicados na virulência de Cryptococcus neoformans são a capacidade de o microrganismo crescer a $37^{\circ} \mathrm{C}$, a presença de uma cápsula polissacarídica rica em glicoxilomanana (GXL), a produção de melanina, a secreção de proteinases e fosfolipases extracelulares, a produção de manitol, urease, assim como o sistema de a mating-type (CASALI et al., 2003). O Cryptococcus neoformans não é um membro da microbiota normal de humanos ou animais (MITCHELL; PERFECT, 1995). É adquirido do meio ambiente através da inalação de leveduras e (ou) basidiosporos, dependendo do estado imunológico do hospedeiro (SOARES et al., 2005). A alta prevalência do Cryptococcus neoformans na natureza e a freqüência relativamente baixa de criptococcose decorre do fato muitas pessoas estarem provavelmente expostas sem desenvolver os sintomas da doença. Os poucos casos relatados de testes cutâneos em humanos com o uso de antígenos ou anticorpos em pessoas saudáveis têm indicado um nível variável de reatividade e exposição ao Cryptococcus neoformans (MITCHELL; PERFECT, 1995).

Sem dúvida, a AIDS trouxe um novo perfil para a prevalência dessa doença, uma vez que a criptococose é uma das mais freqüentes infecções sistêmicas oportunistas (CASALI et al.,
2003). O presente estudo tem o objetivo de realizar uma revisão sistemática da literatura científica dos casos de criptococose ocorridos no Brasil entre 1995-2005.

\section{MATERIAIS E MÉTODOS}

A identificação dos artigos científicos, mediante uma revisão retrospectiva dos casos de criptococose ocorridos no Brasil entre 19952005 foi feita através da base de dados PubMed (National Library of Medicine), utilisando-se "Cryptococcus neoformans" como palavra-chave. Foram encontrados, na base, 7216 artigos. Fazendo-se uso de busca refinada, foram utilizadas as palavras-chave "Brazil" e "Cryptococcus," obtendo-se 83 publicações brasileiras. Como critério de exclusão, foram retirados os artigos de revisão da literatura que incluíam estudos com apenas amostras de origem ambiental ou animal, sem comentários de casos clínicos em humanos. Nos artigos selecionados, foram relacionadas as seguintes variáveis: autor(es), ano, localidade, número de casos investigados, agente(s) etiológico(s), sinais e sintomas, doença isolada ou associada, resposta imune e desfecho.

\section{RESULTADOS}

De acordo com os resultados obtidos, foram totalizados 13 artigos relacionados às variáveis escolhidas entre os anos de 1995-2005, com ocorrência de 1045 casos no Brasil, incluindose estudos referentes a algumas capitais das cinco regiōes do país (QUADRO 1).

\section{DISCUSSÃO}

A revisão sistemática aponta para presença de estudos de casos de criptococose e de série de casos a partir do ano de 1999. Dos 13 publicações, 10 ocorreram nas regiōes Centro-Oeste, Sul e Sudeste. Apenas dois estudos foram realizados nas regiōes Norte e Nordeste, ou seja, dos 578 casos relatados em âmbito local (estados e municípios), $15,92 \%$ ocorreram nessas 


\begin{tabular}{|c|c|c|c|c|c|c|c|}
\hline Autor/ano & Localidade & $\begin{array}{l}\text { Número } \\
\text { de casos }\end{array}$ & Agente etiológico & Sinais/sintomas & $\begin{array}{l}\text { Doença isolada } \\
\text { ou associada }\end{array}$ & $\begin{array}{l}\text { Resposta } \\
\text { imune }\end{array}$ & Desfecho \\
\hline $\begin{array}{l}\text { Delgado et } \\
\text { al., }(2005)\end{array}$ & Campinas & 100 & $\begin{array}{l}\text { Cryptococcus neoformans } \\
\text { var. neoformans }\end{array}$ & SNC, pulmão & $\begin{array}{l}\text { AIDS(35) e sem } \\
\text { AIDS(10) }\end{array}$ & SI & SI \\
\hline $\begin{array}{l}\text { Barreto de } \\
\text { Oliveira et } \\
\text { al.,(2004) }\end{array}$ & Natal & 9 & Cryptococcus neoformans & meningite & AIDS, candidíase & SI & óbito \\
\hline Silva (2004) & São Paulo & 1 & $\begin{array}{l}\text { Criptococcus neoformans, } \\
\text { oocistos álcool ácido } \\
\text { resistentes (sugestivo de } \\
\text { criptosporidium) }\end{array}$ & Febre e meningite & $\begin{array}{l}\text { AIDS, cadidíase, } \\
\text { linfopenia, } \\
\text { hepatite C }\end{array}$ & $\begin{array}{l}\text { Dosagem de } \\
\text { CD4 }(49 \\
\left.\text { céluas } / \mathrm{mm}^{3}\right)\end{array}$ & SI \\
\hline $\begin{array}{l}\text { Casali et al., } \\
(2003)\end{array}$ & Porto Alegre & 105 & Cryptococcus neuformans & SI & AIDS (93 casos) & SI & SI \\
\hline $\begin{array}{l}\text { Cury et al., } \\
(2003)\end{array}$ & São Paulo & 4 & Cryptococcus & $\begin{array}{l}\text { SNC(1), sepse(2), } \\
\text { pulmão(1) }\end{array}$ & AIDS & Sl & óbito \\
\hline $\begin{array}{l}\text { Fernandes } \\
\text { et al., } \\
(2003)\end{array}$ & Goiás & 60 & $\begin{array}{l}\text { Cryptococcus neoformans } \\
\text { var. neoformans sorotipo A } \\
\text { e var. gatii sorortipo B }\end{array}$ & SI & AIDS & SI & SI \\
\hline $\begin{array}{l}\text { Nishikawa } \\
\text { et } \quad \text { al., } \\
(2003)\end{array}$ & Brasil & 467 & $\begin{array}{l}\text { Sorotipos A, D, B e C, } \\
\text { AD }\end{array}$ & Pulmão e SNC & $\begin{array}{l}\text { AIDS(198 casos) } \\
\text { e sem AIDS( } 48 \\
\text { casos) }\end{array}$ & SI & SI \\
\hline $\begin{array}{l}\text { Menezes et } \\
\text { al., (2002) }\end{array}$ & Fortaleza & 5 & Cryptococcus neoformans & SNC & AIDS & SI & SI \\
\hline $\begin{array}{l}\text { Moraes et } \\
\text { al., (2002) }\end{array}$ & $\begin{array}{l}\text { Belo } \\
\text { Horizonte e } \\
\text { Rio de } \\
\text { Janeiro } \\
\end{array}$ & 49 & $\begin{array}{l}\text { Cryptococcus neoformans } \\
\text { var. gatti e var. neoformans }\end{array}$ & SI & SI & SI & SI \\
\hline $\begin{array}{l}\text { Ohkusu et } \\
\text { al., (2002) }\end{array}$ & Sáo Paulo & 84 & $\begin{array}{l}\text { Cryptococcus neoformans } \\
\text { var. grubbi e var. gatii }\end{array}$ & $\begin{array}{l}\text { Alta prevalência de } \\
\text { meningite }\end{array}$ & $\begin{array}{l}\text { Com AIDS }(61 \\
\text { casos) e sem } \\
\text { AIDS ( } 23 \text { casos) }\end{array}$ & SI & SI \\
\hline $\begin{array}{l}\text { Alves et al., } \\
(2001)\end{array}$ & $\begin{array}{l}\text { Rio Grande } \\
\text { do Sul }\end{array}$ & 82 & $\begin{array}{l}\text { Cryptococcus neoformans } \\
\text { var. neoformans }\end{array}$ & Pulmão, SNC & AIDS (38 casos) & SI & SI \\
\hline $\begin{array}{l}\text { Severo., } \\
\text { Zardo, } \\
\text { Londero } \\
(2001)\end{array}$ & Porto Alegre & 1 & $\begin{array}{l}\text { Criptococcus neoformans } \\
\text { var. gattii }\end{array}$ & $\begin{array}{l}\text { Lesôes cutâneas, } \\
\text { cefaléia, fotofobia, } \\
\text { visão turva, } \\
\text { vômitos, tosse, } \\
\text { perda de peso e } \\
\text { fadiga }\end{array}$ & Sem AIDS & $\begin{array}{l}\text { Paciente } \\
\text { imunocomp } \\
\text { etente }\end{array}$ & Alta \\
\hline $\begin{array}{l}\text { Corrêa et } \\
\text { al., (1999) }\end{array}$ & Belém & 78 & $\begin{array}{l}\text { Cryptococcus neoformans } \\
\text { var. neoformans e var. } \\
\text { gattii }\end{array}$ & $\begin{array}{l}\text { SNC e alteraçôes } \\
\text { pulmonares }\end{array}$ & SI & SI & $\begin{array}{l}\text { Alta (13), } \\
\text { óbito (6), SI } \\
(59)\end{array}$ \\
\hline $\begin{array}{l}\text { Total de } \\
\text { casos }\end{array}$ & & 1045 & & & & & \\
\hline
\end{tabular}

Quadro 1- Revisão sistemática dos casos de criptococose ocorridos no Brasil(1995-2005),Natal, RN, 2005

Nota: SI $=$ Sem Informação; SNC $=$ Sistema Nervoso Central

regiōes. No estudo de Nishikawa e colaboradores (2003), dos 467 casos de criptococose relatados no Brasil de 1987 a 1998, 20,6\% ocorreram nas regiōes Norte e Nordeste.

Uma das possíveis explicações quanto à discrepância em relação aos casos para as diferentes regiōes do Brasil vincula-se a fatores relacionados a aspectos geográficos. O Criptococcus neoformans variedade gattii (sorotipos B e C) ocorre nas regióes de clima tropical e subtropical, como foi verificado no estudo de Nishikawa e colaboradores (2003), no qual se constatou que, na região Nordeste, o sorotipo B foi predominante em $87,5 \%$ de indivíduos sem Aids e, na região sudeste, ocorreu em 47\% dos pacientes nas mesmas condições.

Em indivíduos com AIDS, a criptococose é a sexta co-infecção mais prevalente, superando espécies de Cândida, Pneumonicis carinii, espécies de Mycobacterium, Toxoplasma gondii e Herpes vírus simplex (MENEZES et al., 2002).

Segundo o Boletim Epidemiológico AIDS (2003), a doença teve, a partir de 1998, uma redução da taxa de incidência a cada 100.000 habitantes. Para o Brasil, em 2003, verificou-se uma taxa de 5,5. Esses valores da taxa de incidência são de 2,3, 7,5 e 8,5 para as regiōes Nordeste, Sudeste e Sul, respectivamente. Dos 310310 casos de Aids diagnosticados no Brasil de 1980 a 1993, 84,8\% deles estão concentrados nas regiōes Sul e Sudeste.

Embora a taxa de mortalidade tenha sido reduzida de 9,7 em 1995 para 6,3 em 2003, a AIDS continua a ser a principal responsável pelo aumento de infecções oportunistas (BOLETIM EPIDEMIOLÓGICO AIDS, 2003).

Em 10 casos clínicos, a criptococose foi relatada, associada ou não a casos de Aids. Apenas um estudo não apresentou informações a 
respeito de doença associada ou resposta imunológica do paciente (MORAES; PRÍMOLA; HAMDAN, 2003).

Os casos relatados de criptococose em pacientes com imunossupressão ocorreram exclusivamente em indivíduos com AIDS. Em contrapartida, em outras doenças imunossupressoras, como leucemia crônica, linfomas, sarcoidose ou tratamento extensivo com corticosteróide, não foram encontrados relatos (MITCHELL; PERFECT, 1995).

A proporção de casos de criptococose em pacientes com AIDS, quando comparado aos imunocompensados, é, em média, três vezes maior. Isso pode ser visto no estudo de Ohkusu e colaboradores (2002), realizado em São Paulo. Dos 84 pacientes, 61 deles eram portadores do vírus, enquanto 23 não o eram. Já Nishikawa e colaboradores (2003), em um estudo realizado no Brasil, encontraram 198 casos em pacientes com AIDS e 48 sem a doença. Delgado e colaboradores (2005) em Campinas encontraram 35 pacientes com e 10 sem Aids.

Os casos de criptococose relatados em pacientes imunocompensados estão relacionados a áreas geográficas endêmicas para o agente etiológico Criptococcus neoformans gattii. No estudo de Corrêa e colaboradores (1999), as espécies foram identificadas em 19 dos 78 casos ocorridos em Belém, uma vez que o estudo se propôs a investigar a ocorrência em crianças. Oito casos foram de Criptococcus neoformans gattii. $\mathrm{O}$ autor relacionou a patologia com a migração das crianças de regiōes cosmopolitas para áreas rurais e ao desmatamento da floresta amazônica.

Um relato de caso que mereceria uma investigação mais detalhada quanto às variáveis imunológicas e às implicaçōes epidemiológicas é o estudo de Severo, Berta e Zardo e Londero (2001), que relatou um caso clínico de paciente adulto jovem, imunocompensado e infectado pela variedade gattii, na cidade de Porto Alegre.

Os relatos em relação aos sinais e sintomas foram encontrados em nove estudos. Neles, verifica-se que houve comprometimento pulmonar e do Sistema Nervoso Central (SILVA, 2004; CURY et al., 2003; SEVERO;
BERTA E ZARDO; LONDERO, 2001; MENEZES et al., 2002; OHKUSU et al., 2002; NISHIKAWA et al., 2003; ALVES et al., 2001; DELGADO et al., 2005; CORRÊA et al., 1999).

A criptococose é iniciada no pulmão, após inalação da levedura de Criptococcus neoformans. Quando nos espaços alveolares, as leveduras são inicialmente confrontadas com os macrófagos, podendo disseminar-se sistemicamente para o tecido ósseo, a pele, a próstata, com predileção pelo SNC. A interação primária entre o macrófago alveolar e a célula da levedura inalada pode ser um fator determinante para saber se a doença irá desenvolver-se ou não (MITCHELL; PERFECT, 1995).

Os relatos de casos encontrados na literatura apresentam dados restritos quanto ao desfecho da criptococcose. No estudo de Cury e colaboradores (2003), realizado com pacientes com AIDS, todos foram a óbito. No estudo de Severo, Berta e Zardo e Londero (2001), em um paciente imunocompensado, o desfecho foi à alta. No estudo de Corrêa e colaboradores (1999), seis crianças foram a óbito, enquanto dez obtiveram alta sem reinternação e três obtiveram alta com reinternação. Possivelmente, a deficiência em relação ao prognóstico dos pacientes que apresentam criptococcose deve-se ao fato de a grande maioria dos casos ocorrerem em pacientes com Aids, que recebem tratamento para a criptococose. O tratamento com antiretrovirais pode aumentar a resposta imunológica, ao suprimir a replicação viral (HOGAN; HAMMER, 2001) e, conseqüentemente, a infecção oportunista poderá ser controlada sem que o desfecho final seja a morte.

\section{CONSIDERAÇŌES FINAIS}

A revisão de literatura realizada aponta para a AIDS como condição agravante para aumentar a prevalência da criptococose, principalmente nos grandes centros urbanos do Brasil. Ocorre especialmente nas regiōes sul e sudeste, o que implica o aumento do número de internaçôes hospitalares e seqüelas sistêmicas. 


\title{
Cryptococcus neoformans: systematic review of the cases occurred in Brazil
}

\begin{abstract}
The paper performs a systematic revision of clinical cases of patients with cryptococcosis in Brazil. We selected 13 papers printed in PUBMED, from 1995 to 2005. Nine papers refer to clinical cases in regions Central-East, South and Southeast, and three in regions North and Northeast. We studied the several authors, year, locality, number of clinical cases, agent, signals and symptoms, correlated diseases, immune response and outcome. The major cases of cryptococcosis in regions South, Southwest and Central-East occurred in patients with Aids. The cases of Criptococcus neoformans gattii occurred mainly in Northeast. The signals and symptoms of the infection are related to problems in central nervous system and lungs. The outcome of cryptococcosis cases described in the literature is reduced probably due to co-infection with Aids.
\end{abstract}

Keywords: Cryptococcus neoformans; cryptococcosis - Review Literature.

\section{REFERÊNCIAS}

ALVES, S.H. et al. In vitro susceptibility to antifungal agents of clinical and enviromental Cryptococcus neoformans isolated in southern of Brazil. R. Inst. Med. Trop. S. Paulo, São Paulo, v.43, n.2, p.267-270, 2001.

BOEKHOUT, T. Theelen et al. Hybrids genotypes in pathogenic yeast Cryptococcus neoformans. Mycrobiology, Reading, v.147, p.891-907, 2001.

BOLETIM EPIDEMIOLÓGICO AIDS. Brasília, DF: Ministério da Saúde, ano 17, n.1, jan./dez. 2003. 56p.

CASALI, A.K .et al. Molecular typing of clinical and environmental Cryptococcus neoformans isolates in Brazilian state Rio Grande do Sul. FEMS Yeast Research, Amsterdam, v.3, p.405415, 2003.

CORRÊA, M.P.S.C. et al. Criptococose em crianças no Estado do Pará, Brasil. R. Soc. Bras. Med. Trop., Rio de Janeiro, v.32, n.5, p.505508, set./out. 1999.

CURY, P.M. et al. Autopsy findings in AIDS patients from a reference hospital in Brazil: analysis of 92 cases. Pathol. Res. Pract., Sttutgart, v.199, p.811-814, 2003.

DELGADO, A.C.N. et al. Human cryptococcosis: relationship of environmental and clinical strains of Cryptococcus neoformans var. neoformans from urban and rural areas. Mycopathologia, Dordrecht, v.159, p.7-11, 2005.

HOGAN, C.M.; HAMMER, S.M. Host determinants in HIV infection and disease. Ann. Internal Medicine, Philadelphia, v.134, p.978-996, 2001.

LENGELER, K.B.; COX, G.M.; HEITMAN, J. Serotype AD strains of Cryptococcus neoformans are diploid or aneuploid and are heterozygous at the mating type locus. Infect.Immun., Washington, DC, v.69, p.115122, 2001.

MENEZES, E.A. et al. Cryptococcus neoformans causing meningitis in AIDS patients. R. Soc. Bras. Med. Trop., Rio de Janeiro, v.35, n.5, p.537-539, set./out. 2002.

MITCHELL, T.G.; PERFECT, J.R. Cryptococcosis in the era of AIDS: 100 years after the discovery of Cryptococcus neoformans. Clin. Microbiol. Rev., Washington, DC, v.8, n.4, p.515-548, Oct.1995.

MORAES, E.M.; PRÍMOLA, N.S.; HAMDAN, J.S. Antifungal susceptibility of clinical and environmental isolates of Cryptococcus neoformans to four antifungal drugs determined by two 
techniques. Mycoses, Berlin, v.46, p.164-168, 2003.

NISHIKAWA, M.M. et al. Serotyping of 467 Cryptococcus neoformans isolates from clinical and environmental sources in Brazil: analysis of host and regional patterns. J. Clin. Microbiol., Washington, DC, v.41, n.1, p.73-77, 2003.

OHKUSU, M. et al. Serotype, mating type and ploidy of cryptococcus neoformans strains isolated from patients in Brazil. R. Inst. Med. Trop. S. Paulo, São Paulo, v.44, n.6, p.299-302, nov./dez. 2002.

OLIVEIRA, M.T. Barreto de et al. Cryptococcus neoformans shows a remarkable genotypic diversity in Brazil. J. Clin. Microbiol., Washington, DC, v.42, n.3, p.1356-1359, 2004.

SEVERO, L.C.; BERTA E ZARDO, I.B.; LONDERO, A.T. Cutaneos crytococcosis due to Cryptococcus neoformans var. gattii. R. Iberoam. Micol., Bilbao, v.18, p.200-201, 2001.

SILVA, R.M.G. Meningite por Cryptococcus neoformans como causa da febre prolongada em paciente com AIDS. R. Assoc. Méd. Bras., São Paulo, v.50, n.2, p.109-126, 2004.

SOARES, M.C.B. et al. Enviromental strains of Cryptococcus neoformans variety grubii in the city of Santos, SP, Brazil. R. Inst. Med. Trop. S. Paulo, São Paulo, v.47, n.1, p.31-36, 2005.

SORREL, T. et al. Natural environmental sources of Cryptococcus neoformans var. gattii. J.Clin.Microbiol., Washington, DC, v.34, p.1261-1263, 1996.

VERONESI, R.; FOCACCIA, R. Tratado de infectologia. Rio de Janeiro: Atheneu, 1996. 